\title{
Références bibliographiques du dossier «L'école dans les médias »
}

\section{Bernadette Plumelle}

\section{OpenEdition}

12 Journals

Édition électronique

URL : http://journals.openedition.org/ries/4011

DOI : $10.4000 /$ ries.4011

ISSN : 2261-4265

Éditeur

Centre international d'études pédagogiques

\section{Édition imprimée}

Date de publication : 1 septembre 2014

Pagination : 109-120

ISBN : 978-2-85420-604-3

ISSN : 1254-4590

Référence électronique

Bernadette Plumelle, "Références bibliographiques du dossier « L'école dans les médias » », Revue internationale d'éducation de Sèvres [En ligne], 66 | septembre 2014, mis en ligne le 01 septembre 2014, consulté le 22 juin 2020. URL : http://journals.openedition.org/ries/4011 ; DOI : https://doi.org/ 10.4000/ries.4011 


\title{
Références \\ bibliographiques
}

\section{Bernadette Plumelle}

La littérature sur le traitement des questions scolaires par les médias est principalement anglosaxonne. Constatant la faible présence de travaux francophones, nous avons choisi de présenter, pour cet article, des références récentes, extraites pour la plupart de la base de données bibliographiques américaine ERIC (Education resources information center). Les résumés sont basés sur ceux des éditeurs. La sélection, établie avec l'aide précieuse de Xavier Pons, a visé à proposer la plus grande couverture géographique possible et à donner un aperçu des grandes thématiques débattues dans les médias, telles que l'enquête PISA, la représentation des enseignants, les palmarès des établissements scolaires. Les deux premières parties proposent une sélection de références sur 1) les influences réciproques des médias et des politiques publiques en éducation ; 2) des approches comparatives de couvertures médiatiques de l'éducation. Cette bibliographie est conçue comme un complément aux références bibliographiques données dans les articles du dossier "l'école dans les médias »; ces dernières ne sont pas reprises ici, à l'exception de quelques références anciennes et de celles sur la France.

Bibliographie arrêtée le 26 août 2014.

\section{MÉdIAS ET POLITIQUES PUBLIQUES EN ÉDUCATION : INFLUENCES RÉCIPROQUES}

\begin{abstract}
AMSING Hilda T. A., GREVELING Linda, DEKKER Jeroen J. H., " The struggle for comprehensive education in the Netherlands: the representation of secondary school innovation in Dutch newspaper articles in the 1970s ", History of Education, 2013, vol. 42, $n^{\circ}$ 4, p. 460-485

Cet article porte sur la façon dont les journaux néerlandais ont présenté le débat sur l'enseignement secondaire général aux Pays-Bas dans les années 1970 et influencé l'expérimentation de la "middle school » néerlandaise. L'article montre les différents rôles joués par les journaux, celui de diffuseur de nouvelles idées pour susciter le débat, de communicateur des vues politiques et d'influence sur les stratégies politiques choisies.
\end{abstract}

ANDERSON Gary L., " Media's impact on educational policies and practices: political spectacle and social control ", Peabody journal of education, 2007, vol. $82, n^{\circ} 1$, p. 103-120

Les médias utilisent la mise en spectacle pour susciter des points de vue, des perceptions, des angoisses, des aspirations et renforcer ou saper le soutien à des politiques éducatives spécifiques, des pratiques et des idéologies. Cet article explore les mécanismes par lesquels ce spectacle est construit, avec un accent particulier sur le rôle des médias et des politiques et pratiques de la réforme de l'école. Enfin, les implications sur la façon dont le pouvoir fonctionne dans le cadre de la mise en spectacle des médias sont passées en revue, ainsi que les implications pour les praticiens de l'école et de sa réforme. 
CABALIN Christian, "The conservative response to the 2011 Chilean student movement: neoliberal education and media ", Discourse: studies in the cultural politics of education, 2014, vol. $35, n^{\circ} 4$, p. 485-498

Cet article présente une analyse critique du discours des éditoriaux publiés sur le mouvement étudiant de 2011 dans le journal El Mercurio, journal conservateur très influent au Chili. El Mercurio a construit le débat public sur les politiques éducatives et défendu l'éducation néolibérale en se basant sur trois discours : le système néolibéral est parfait (absolute), l'enseignement public obtient de moins bonnes évaluations que l'enseignement privé et l'éducation est une question technique et non politique.

FAIRBROTHER Gregory P., «Forging consensus for implementing youth socialization policy in Northwest China ", International Journal of Educational Development, mars 2011, vol. 31, $n^{\circ}$ 2, p. 179-186

L'article examine le rôle joué en Chine par les médias de l'éducation produits dans les provinces chinoises pour l'établissement d'un consensus entre les acteurs locaux responsables de la mise en ouvre de la nouvelle politique de socialisation des jeunes promulguée au niveau national.

FORESTIER Yann, "Remettre l'éducation à la une des journaux". Bilan des années Allègre (1997-2000)». Vingtième Siècle. Revue d'histoire, 2007, n 94, p. 231-242. [http://goo.gl/utFLpl]

La volonté du ministre de l'éducation Claude Allègre d'animer le débat scolaire dans le but de conduire des réformes ambitieuses a été mise à l'épreuve lors de la genèse de la réforme des lycées (1998-1999). Renonçant à profiter de la dynamique créée par la consultation préliminaire et négligeant les soutiens qu'il aurait pu trouver, il a laissé les adversaires de la réforme définir les termes du débat. Développant la dénonciation d'un complot "pédagogiste » qui préparerait la destruction de l'institution scolaire, leur discours a fourni à l'opposition multiforme rencontrée par Claude Allègre un fondement idéologique qu'on peut désigner sous le nom d'« antipédagogisme».

LEVIN Ben, " Media-government relations in education », Journal of education policy, mai 2004, vol. 19, $n^{\circ} 3$, p. 271-283

Le gouvernement et les médias sont intimement liés à bien des égards même si des relations de méfiance existent. Cet article, basé sur l'expérience gouvernementale de l'auteur, décrit les mesures que les gouvernements tentent de mettre en place avec les médias et aborde trois principales plaintes au sujet de la couverture médiatique : sa tendance à simplifier, à jeter le blâme, et principalement à être axée sur le court terme.

LINGARD Bob, RAWOLLE Shaun, " Mediatizing educational policy: the journalistic field, science policy, and cross-field effects ", Journal of Education Policy, 2004, vol. 19, $n^{\circ} 3$, p. 361-380

Cet article a pour objectif de démontrer l'utilité de la théorie de Bourdieu, y compris les concepts de champ, des logiques de pratique et d'habitus, pour comprendre les relations entre les médias et la politique, en l'illustrant par une étude de cas récente de la politique australienne. L'étude montre aussi les effets de cette politique en matière d'éducation, illustrant la façon dont, aujourd'hui, la politique éducative peut naître de l'évolution d'autres domaines de politique publique. 
OPFER Darleen $V_{\text {., }}$ "Developing a research agenda on the media and education ", Peabody journal of education, 2007, vol. 82, $n^{\circ}$ 1, p. 166-177 [http://goo. gl/JCZWtN ]

La plupart des chercheurs qui ont étudié la relation entre les médias et l'éducation ont affirmé que la couverture de l'éducation a un impact négatif sur la perception du public et le soutien à l'enseignement public. Une grande partie de cette recherche s'appuie sur des exemples de cas négatifs et des données transversales pour faire cette demande. Cet article remet en question les hypothèses formulées par ces chercheurs et suggère l'élaboration d'un cadre qui reconnaît le rôle approprié des médias dans une société démocratique.

STACK Michelle L., "Spin as Symbolic Capital: The Fields of Journalism and Education Policy-Making ", International journal of leadership in education, avril 2010, vol. 13, $n^{\circ}$ 2, p. 107-119

Comment les décideurs politiques et les journalistes accumulent-ils du capital symbolique en termes de positionnement stratégique par rapport à l'élaboration des politiques? Comment ont-ils accès à l'information concernant l'éducation et la scolarisation et comment celle-ci est-elle construite? L'article analyse dix-sept entretiens avec d'anciens ministres canadiens de l'éducation, premiers ministres, bureaucrates et journalistes chevronnés. Cet article soutient que le journalisme et l'élaboration des politiques d'éducation ont des points de convergence. L'étude conclut que les questions de cycles électoraux et de l'actualité journalistique et la politique de reconnaissance dans les domaines du journalisme et de la politique gouvernementale d'éducation sont essentielles pour la compréhension de la politique éducative.

SUNG Youl-Kwan, KANG Mi Ok, "The cultural politics of national testing and test result release policy in South Korea: a critical discourse analysis ", Asia Pacific Journal of Education, 2012, vol. 32, $n^{\circ}$ 1, p. 53-73

L'article examine la construction idéologique du discours éducatif dans la presse écrite sudcoréenne. De manière significative, ces discours ont récemment promu une très large évaluation nationale et la divulgation des résultats aux tests. Grâce à un examen attentif de cette politique («test plus release»), les auteurs montrent comment le gouvernement a façonné l'opinion publique. S'appuyant sur une analyse critique du discours des textes de politique éducative du gouvernement et sur des sources de la presse écrite, cet article examine comment les discours sur la "responsabilité ", "le droit de savoir " et "l'équité des tests " ont été produits et contextualisés. Les auteurs analysent également comment le gouvernement et les discours influents de la presse écrite interagissent avec des facteurs politiques et culturels afin d'obtenir l'assentiment du public pour la nouvelle politique de «test plus release».

\section{THOMAS Sue, Education Policy in the media: Public discourses on education,} Eumundi: PostPressed, 2006, 385 p.

L'ouvrage offre un compte rendu minutieux de la relation entre les représentations médiatiques de la politique de l'éducation, et le processus par lequel ces politiques sont élaborées. En s'appuyant sur un examen approfondi du curriculum de l'école Queensland (Australie), l'auteure propose une analyse du processus et des résultats de cet examen; elle présente également une analyse détaillée du contexte médiatique dans lequel il a eu lieu ainsi que des enjeux et pressions du public auxquels il a été nécessaire de faire face.

WALLACE Mike, "Discourse of derision: the role of the mass media within the education policy process ", Journal of Education Policy, 1993, vol. 8, $n^{\circ}$ 4, p. 321-337 On affirme que les médias jouent un rôle important dans le processus politique de l'éducation mais cette analyse a reçu peu d'écho dans les travaux empiriques et théoriques. L'auteur définit des concepts qui peuvent aider à cette exploration du rôle des médias, y compris la notion anthropologique de mythe. Ces concepts sont utilisés ici pour l'analyse d'une question 
politique dans laquelle le gouvernement britannique et les médias de masse ont encadré le débat entre éducation progressive et traditionnelle. L'analyse s'appuie sur l'expérience d'une école primaire qui a été dépeinte dans une émission de télévision comme illustrant des pratiques éducatives progressistes.

WOLFF Éliane, "Presse et institution scolaire : une co-construction de l'information sur l'École à la Réunion », Cahiers de la recherche sur l'éducation et les savoirs, 2002, $n^{\circ} 1, p$. 33-55 [http://cres.revues.org/1662]

L'auteure met à jour quelques éléments du processus d'élaboration de l'information sur l'école et les jeunes sur l'île de la Réunion. Une rapide évocation du paysage médiatique et scolaire réunionnais rappelle le contexte spécifique d'une ancienne colonie française devenue département il y a moins de cinquante ans et traversée depuis par de profondes et massives mutations. Les résultats partiels donnés ici tentent de saisir la façon dont des journalistes spécialisés en éducation des deux principaux quotidiens) et leurs interlocuteurs institutionnels majeurs (des fonctionnaires chargés de la communication au rectorat de la Réunion) co-construisent le discours médiatique sur l'école.

\section{APPROCHES COMPARATIVES DES COUVERTURES MÉDIATIQUES DE L'ÉDUCATION}

BIANCHINI Pascal, "Entre instrumentalisation et autonomisation. Journalistes et militants dans les luttes scolaires et universitaires au Sénégal et au Burkina Faso (années soixante - quatre-vingt-dix)», Cahiers de la recherche sur l'éducation et les savoirs, 2002, $n^{\circ} 2$, p. 151-178

En Afrique subsaharienne, l'histoire de la presse et celle des mouvements sociaux issus de la crise de l'enseignement ont été caractérisées par un niveau élevé d'interdépendance. Les journaux constituent un miroir des différentes mobilisations sociales autour des enjeux scolaires et universitaires, à travers des stratégies plus ou moins élaborées de censure et de dévoilement. Mais le développement de nouvelles formes de presse est en partie l'effet de l'existence de ces mouvements sociaux qui ont contesté l'hégémonie de la classe dirigeante. La " configuration communicationnelle » qui en résulte n'est cependant pas univoque : le cas du Sénégal permet d'entrevoir les premières étapes de la constitution d'un "champ journalistique » autonome, tandis que, dans le cas du Burkina Faso, la forte polarisation socio-politique a fortement perturbé cette dynamique institutionnelle.

GÉRARD Etienne, POTEAU Laurence (coordinateurs), "Écoles et “jeunes" dans les médias du sud: dossier ", Cahiers de la recherche sur l'éducation et les savoirs, 2002, $n^{\circ}$ 2, 316 p. [http://cres.revues.org/1546]

Ce dossier thématique interroge un espace singulier de production de discours sur l'École et les «jeunes", celui de la presse écrite en Afrique (Algérie, Côte d'Ivoire, Congo, Maroc, Sénégal, etc.). Chaque article du dossier les aborde à sa manière et à la lumière de son propre terrain. Quelle place occupent l'éducation scolaire et la «jeunesse» dans les médias des pays du «Sud»? Quelles sont les logiques qui président à la sélection, à la construction et au traitement de l'information dans ces deux domaines politiquement et socialement sensibles?

\section{HARLÉ Mélusine, École et télévision : le choc des cultures, Paris : L'Harmattan, 2004, 194 p.}

En entrant dans le cœur de la représentation de l'école dans les journaux télévisés français et américains, on comprend assez vite que l'actualité ne rend pas compte de la réalité, mais d'un regard sur cette dernière, regard influencé par les pratiques journalistiques françaises 
et américaines, par un régime de croyances. La seule clé de lecture de ces représentations du réel est alors à chercher du côté du mythe de l'école dans nos sociétés occidentales et d'un imaginaire utilitariste et déshumanisé véhiculé dans nos médias et dans nos sociétés respectives.

PONS Xavier, "Going beyond the "PISA Shock" Discourse: An Analysis of the Cognitive Reception of PISA in Six European Countries, 2001-2008 », European Educational Research Journal, 2012, vol. 11, $n^{\circ}$ 2, p. 206-226

L'objet de cet article est d'analyser la réception cognitive de Pisa dans six pays européens qui ont été étudiés dans le cadre du projet de recherche européen Know\&Pol (Knowledge and Policy in Education and Health Sectors). La réception ne désigne pas ici une simple transmission verticale de messages du niveau international au niveau national mais une activité cognitive au cours de laquelle ces messages sont continuellement acceptés, refusés ou renégociés. L'article se concentre sur les aspects cognitifs de ce processus incessant de redéfinition et tente de comprendre le type de connaissances que les acteurs mobilisent dans le débat public au nom de Pisa, dans quels contextes et pour quelles raisons. Pour cela, l'auteur propose un cadre d'analyse spécifique qui emprunte largement à la sociologie de la traduction et à son adaptation à l'analyse des politiques publiques.

TAKAYAMA Keita, WALDOW Florian, SUNG Youl-Kwan, "Finland has it all? Examining the media accentuation of "Finnish education" in Australia, Germany and South Korea", Research in Comparative and International Education, 2013, vol. 8, $n^{\circ}$ 3, p. 307-325

L'article examine la façon dont la presse écrite met en exergue "l'éducation finlandaise » (Finnish education) et l'insertion de cette référence dans les discours politiques sur la réforme de l'éducation en Australie, en Allemagne et en Corée du Sud. L'étude identifie tous les articles faisant référence à "l'éducation finlandaise » publiés de 2000 à 2011 dans deux journaux à grand tirage de chaque pays avec des orientations politiques différentes. L'analyse du discours montre les différentes façons dont "l'éducation finlandaise " est mise en relief par les journaux, servant à légitimer les différents agendas politiques dans les débats de politique éducative. Il fait valoir que "l'éducation finlandaise" est devenue un "écran de projection" des diverses conceptions de "bonne éducation" et les visions associées de «bonne société ».

WALDOW Florian, TAKAYAMA Keita, SUNG Youl-Kwan, "Rethinking the pattern of external policy referencing: media discourses over the "Asian Tigers" PISA success in Australia, Germany and South Korea? ", Comparative education, 2014, vol. 50, $n^{\circ} 3$, p. 302-321

L'article compare la manière dont le succès des "tigres asiatiques" dans l'enquête PISA, en particulier PISA 2009, a été présenté dans les médias en Australie, en Allemagne et en Corée du Sud. Il fait valoir que même à l'époque actuelle de la «globalisation du champ de la politique éducative ", les facteurs locaux sont importants pour déterminer si un pays devient ou non une société de référence pour la réforme éducative. L'article vise à découvrir certains de ces facteurs, à identifier les stéréotypes diffusés à l'échelle mondiale sur l'éducation en Asie, les relations économiques et le sentiment de "crise " provoquée par la position relative et le changement de position des pays en question dans les classements PISA. 


\section{LES TESTS ET PALMARÈS COMME OBJETS MÉDIATIQUES Le traitement de l'enquête PISA dans la presse}

CHAMPOLLION Pierre, BARTHES Angela, « De l'usage et du mésusage du classement par rang en matière de médiatisation de l'évaluation internationale PISA », Questions vives recherches en éducation, 2012, vol. 6, $n^{\circ} 16$, p. 15-27, [http://goo.gl/oxx7Ec]

Si les classements des pays par rang issus des évaluations PISA n'ont qu'une faible signification statistique, leur médiatisation a souvent été mise à contribution, notamment en France, pour essayer de légitimer des mesures et des réformes éducatives. Mais par-delà ce positionnement très médiatique, les évaluations PISA permettent d'avancer un certain nombre de facteurs explicatifs comparatifs relatifs aux aspects organisationnels de l'éducation.

MONS Nathalie, PONS Xavier, "Pourquoi n'y a-t-il pas eu de "choc PISA" en France? Sociologie de la réception d'une enquête internationale (2001-2008) », Revue française de pédagogie, mars 2013, $n^{\circ} 182$, p. 9-17

L'article analyse la réception de l'enquête PISA dans le débat public national en France entre 2001 et 2008, mettant en évidence que les connaissances énoncées dans le débat public au nom de PISA sont les produits des configurations d'action publique à l'œuvre au moment de leur publication. La première partie, fondée sur une enquête qualitative conduite par les auteurs dans le cadre du projet européen Knowlege and Policy, analyse leurs configurations et pouvoir de structuration du débat public. La seconde mobilise les études de cas effectuées dans d'autres pays européens dans le cadre de ce projet pour interroger les éventuelles spécificités du débat public français sur PISA.

PONS Xavier, "Qu'apprend-on vraiment de Pisa? Sociologie de la réception d'une enquête internationale dans trois pays européens (2001-2008) », Revue internationale d'éducation de Sèvres, septembre 2010, p. 51-59 [http://ries. revues.org/85]

Synthétisant les résultats d'une recherche collective internationale (projet KnowePol), cet article interroge les effets du programme international de suivi des acquis des élèves (PISA) de l'OCDE sur le débat public dans trois pays européens (Écosse, France et Portugal). Mobilisant un cadre théorique propre à la science politique, il montre que PISA n'a pas permis un apprentissage politique de la part des acteurs des systèmes éducatifs concernés mais a eu pour principal effet de confirmer, en les légitimant par le sceau d'une enquête scientifique, un ensemble d'opinions et de positions politiques qui lui préexistaient.

SELLAR Sam, LINGARD Bob, "Looking East: Shanghai, PISA 2009 and the reconstitution of reference societies in the global education policy field", Comparative education, 2013, vol. 49, $n^{\circ} 4$, p. 464-485

L'article examine la performance exceptionnelle de Shanghai, en Chine dans l'enquête PISA 2009 et ses effets sur les autres systèmes nationaux et dans le domaine de la politique éducative. Les effets de la réussite de Shanghai sont considérés dans trois contextes nationaux : aux États-Unis, en Angleterre et en Australie. Les auteurs combinent (a) l'analyse de données de plus de trente entretiens avec des acteurs politiques de haut niveau de l'OCDE, de l'IEA (International Association for the Evaluation of Educational Achievement), en Australie et en Angleterre; (b) l'analyse des documents de discours politiques, des rapports de recherche 
commandés et de la couverture médiatique des trois contextes nationaux. La performance de Shanghai au PISA 2009 a repositionné ce système comme une nouvelle "société de référence ", déplaçant le regard global de l'éducation de la Finlande vers l'Orient.

TAKAYAMA Keita, "The politics of international league tables: PISA in Japan's achievement crisis debate", Comparative Education, 2008, vol. 44, $n^{\circ} 4$, p. 387-407

L'article analyse le rôle des acteurs, en particulier celui des journaux nationaux et du ministère de l'éducation, dans la pression sur la politique curriculaire exercée par les classements de l'enquête PISA. Utilisant le récent débat de politique éducative au Japon comme étude de cas, l'auteur montre comment les médias japonais ont interprété les résultats du PISA 2003 d'une manière qui entre en résonance avec le contexte culturel, politique et économique spécifique de l'époque et analyse la manière dont le ministère de l'éducation a utilisé les résultats pour légitimer des mesures de politique très controversées.

\section{Autres palmarès}

BOUCHARD Julie, "Le classement : instrument de valorisation des universités parisiennes ", Hermès La Revue, février 2013, n 66, p. 199-205

Dans quelle mesure et comment les classements des établissements d'enseignement supérieur et de recherche affectent-ils la "présentation de soi » des universités à leurs publics ? L'article s'inspire de la sociologie des sciences et des techniques et de la quantification, voyant dans le classement un "instrument ", c'est-à-dire une "institution sociale », possédant des propriétés irréductibles. Il analyse ensuite la présence des classements sur les sites web des universités parisiennes liés à la mise en valeur des universités et les motifs discursifs récurrents que ces classements induisent.

ELSTAD Eyvind, "Schools which are named, shamed and blamed by the media: school accountability in Norway", Educational Assessment, Evaluation and Accountability, mai 2009, vol. 21, $n^{\circ}$ 2, p. 173-189

Depuis que la Norvège a introduit des tests et d'autres types d'évaluations, la presse a mis l'accent de manière négative sur les écoles qui ont eu de mauvaises performances. La presse reconstruit à partir des sources publiques des classements (league tables) des résultats des élèves, alors que la position officielle s'oppose au classement public des écoles. Cet article traite des conséquences de cette couverture médiatique négative dans les écoles. Ce coup de projecteur des médias sur ces écoles met en jeu un processus émotionnel complexe, qui peut inciter les écoles à des mécanismes d'amélioration, mais aussi provoquer des réactions hostiles ou paniquées.

MOCKLER Nicole, "Reporting the "Education revolution": My school.edu.au in the print media ", Discourse: studies in the cultural politics of education, 2013, vol. 34, $n^{\circ} 1$, p. 1-16 [http://goo.gl/bj1WvF]

Le site MySchool.edu.au, lancé en janvier 2010, a fait l'objet d'une intense couverture médiatique. Il classe et compare les écoles selon les résultats aux tests standardisés de littératie et de numératie. L'article examine 34 éditoriaux axés sur MySchool et identifie trois récits principaux en fonctionnement, ceux de la méfiance, du choix et de la performance. Il fait valoir que ces récits travaillent ensemble pour renforcer et promouvoir le discours éducatif néolibéral. Les récits dominants générés par le site et les classements publiés par les journaux se présentent comme la solution aux problèmes de faible performance, des «mauvaises » écoles et des «mauvais » enseignants. 
MURPHY Roger, "Media Roles in Influencing the Public Understanding of Educational Assessment Issues ", Oxford Review of Education, 2013, vol. 39, $n^{\circ}$ 1, p. 139-150

Cet article explore la couverture médiatique des résultats de l'examen national au RoyaumeUni. L'article s'appuie sur les conclusions d'une étude précédente portant sur la publication de résultats du certificat général de l'enseignement secondaire et du certificat général de niveau avancé qui avait révélé l'utilisation de certains « modèles médiatiques " standards conduisant à une gamme assez prévisible de reportages, quelle que soit la tendance des résultats de l'année. Ce document examine les implications plus larges du rôle des médias pour influencer le public sur les questions d'évaluation.

RAPTIS Helen, "Ending the reign of the Fraser institute's school rankings ", Canadian journal of education, 2012, vol. 35, $n^{\circ} 1$, p. 187-201 [http://goo. $\mathrm{gl} / 15 \mathrm{Rjd} \mathrm{A}]$

Le classement des écoles par l'Institut Fraser est très populaire auprès des parents et de la presse. Depuis plus d'une décennie, ces classements ont été particulièrement pénalisants pour les écoles mal classées, dans des secteurs de faible niveau économique et de forte pauvreté, quand les parents d'enfants qui réussissent bien à l'école décident de les changer d'établissement pour un autre mieux classé. En février 2010, après avoir défendu le droit des parents d'avoir accès à ce classement, un journal de Victoria, le Times-Colonist, a décidé de ne pas les publier. L'article s'appuie sur l'analyse critique du discours pour explorer le long règne de ces classements dans les médias et la décision soudaine du Times-Colonist d'arrêter leur publication.

\section{LA REPRÉSENTATION DES ENSEIGNANTS DANS LES MÉDIAS}

COHEN Jennifer L., "Teachers in the news: a critical analysis of one US newspaper's discourse on education, 2006-2007 ", Discourse: studies in the cultural politics of education, 2010, vol. $31, n^{\circ} 1$

Le discours sur l'enseignement public aux États-Unis a été caractérisé par des messages de crise. Les politiques éducatives identifient le manque d'enseignants qualifiés et le manque de contrôle des pratiques enseignantes comme étant des facteurs de la crise. Les grands médias d'information sont des lieux essentiels pour le débat politique sur l'éducation. L'auteur utilise l'analyse critique du discours pour étudier le discours tenu sur les enseignants dans un grand quotidien américain.

GOLDSTEIN Rebecca A., "Imaging the frame: media representations of teachers, their unions, NCLB, and education reform ", Educational policy, juillet 2011, vol. $25 n^{\circ}$ 4, p. 543-576

Cet article porte sur le discours politique entourant la réforme éducative No child left behind (NCLB) et montre comment ce discours a modelé la perception de l'enseignement public durant l'administration Bush. En s'appuyant sur les campagnes médiatiques dans le New York Times et le Time Magazine, l'article montre comment les médias ont visuellement et textuellement encadré et renforcé la loi NCLB et les réformes comme étant la seule solution pour remédier aux défaillances de l'enseignement public et en attaquant les syndicats d'enseignants et les enseignants. L'analyse révèle que les médias ont présenté une image extrêmement négative des syndicats d'enseignants, comme des opposants à NCLB et aux tentatives de réforme de l'école. 
HANSEN Anders, "Researching "teachers in the news": The portrayal of teachers in the British national and regional press ", Education, 2009, vol. 37, $n^{\circ} 4$, p. 335-347

L'auteur présente les résultats d'une étude approfondie sur la représentation des enseignants et de l'éducation dans la presse. En mettant l'accent sur la représentation des enseignants dans les gros titres des articles, l'étude montre un changement lexical et syntaxique considérable de la représentation des enseignants dans la presse entre 1991 et 2005, passant d'un point de vue négatif sur les enseignants à une expression plus positive de la profession enseignante présentée comme travaillant beaucoup et devant faire face à des pressions croissantes.

PUN Sydney S., "The politics of hope and cynicism in the realization of the vision of the "334" education reform in Hong Kong", Asia Pacific Education Review, mars 2013, vol. 14, $n^{\circ} 1$, p. 55-65

À Hong-Kong, la mise en place d'une politique de l'éducation considérée comme la plus importante de ces dernières années semblait acceptée dans son principe, les points d'achoppement résidant dans le calendrier et les modalités d'application. Mais les suicides de deux enseignants ont provoqué une vague de protestations sans précédent contre la politique éducative du gouvernement. Cet article vise à donner un sens à ces événements à travers une analyse critique du discours de la politique éducative, avec des matériaux tirés de documents, de discours et de communiqués de presse publiés par le gouvernement ainsi que des articles du journal South China Morning Post.

RACHAEL Gabriel, LESTER Jessica Nina, « The Romance Quest of Education Reform: A Discourse Analysis of the Los Angeles Times' Reports on Value-Added Measurement Teacher Effectiveness ", Teachers college record, 2013, vol. 115, $n^{\circ} 12,32$ p.

L'article montre comment les médias, particulièrement le journal The Los Angeles Times, sont entrés dans le débat concernant l'évaluation des enseignants, avec pour résultat un récit qui a façonné la manière dont le public perçoit l'efficacité des enseignants. En 2010, le journal a pris pour sujet la place et la valeur de la mesure de la valeur ajoutée (value-added measurement), en faisant valoir qu'il s'agit d'un outil pour supprimer les enseignants présumés inefficaces. Les recherches portant sur la politique de l'éducation sont souvent représentées par les médias de façon à amener le public à prendre des positions spécifiques et à attribuer la responsabilité à des groupes particuliers. À partir d'une analyse de discours effectuée sur 52 articles publiés entre 2009 et 2011, l'auteur porte une attention particulière à la manière dont le discours médiatique a fonctionné pour politiser et simplifier les questions liées à l'évaluation des enseignants.

REIMERS Eva, "Discourses of education and constitution of class: public discourses on education in Swedish PBS television ", Discourse: studies in the cultural politics of education, 2014, vol. $35, n^{\circ} 4$, p. 540-553

L'article examine le discours éducatif public en Suède. Il montre comment un discours dominant néolibéral sur l'éducation est articulé en même temps à un discours sur l'égalité des chances en éducation, les deux discours s'influençant et se subvertissant de sorte qu'aucun n'est hégémonique. Il prend pour point de départ l'accent néolibéral mis sur l'individu, en particulier en ce qui concerne le choix de l'école et l'importance de la classe pour la réussite scolaire.

THOMAS Sue Allan, "Newspapers on education policy: constructing an authoritative public voice on education" in Fitzgerald, R. \& Housley, W. (Eds.) Media, Policy and Interaction, London : Ashgate Publishing, 2009, p. 205-223 [http://goo.gl/bb3SoH]

À partir d'un corpus d'articles de presse du journal national The Australian, l'auteure analyse la façon dont le journal construit les catégories d'enseignement et d'enseignant "bon » et «mauvais» dans le contexte de la politique mise en place par les autorités fédérales et son programme Australian Government Quality Teacher Programme (AGQTP). Elle explore les 
relations entre les médias et le programme AGQTP mis en place par le ministère. Elle examine ensuite les contributions des membres du gouvernement fédéral à ces débats et montre comment une analyse critique du discours peut fournir un cadre pour la compréhension et l'exploration des liens entre les médias et la politique éducative du gouvernement.

\section{LA CRISE DE L’ÉCOLE DANS LES MÉDIAS}

BALLAND Ludivine, Une sociologie politique de la crise de l'école : de la réussite d'un mythe aux pratiques enseignantes, thèse de doctorat de science politique de l'université-Paris-Ouest-Nanterre-La Défense, 2009, 2 vol., 695 p.

La thématique de la crise de l'école est aujourd'hui omniprésente dans les discours publics. Si l'on peut attester de l'existence de ces nombreux discours à différentes périodes, le début des années 1980 est décisif car il constitue un moment de solidification et de politisation de cette doxa. À partir de ces années charnières, cette thèse se propose d'examiner les différents niveaux de formulation de la «crise de l'École » : le débat public (l'espace des livres publiés sur l'école, émissions télévisées, presse écrite) ; le champ politique et en particulier, le rôle des cabinets ministériels dans le travail de la consécration étatique de la crise ; les établissements scolaires enfin, pour apprécier aujourd'hui la manière dont les enseignants se positionnent, reprennent on non cette vision dominante de leur métier.

BERLINER David, BIDDLE Bruce, The manufactured crisis: Myths, fraud, and the attack on America's public schools, White Plains, New York: Longman, 1997, 432 p.

De très nombreuses critiques se sont développées sur l'état supposé des écoles publiques américaines. Les enfants n'apprennent pas ce qu'ils devraient, la violence et le chaos règnent dans la salle de classe, et la bureaucratie étrangle les tentatives de réforme. Mais dans quelle mesure cette image sombre reflète-t-elle la vérité ? Les auteurs démystifient des statistiques familières mais fausses sur les écoles publiques concernant la soit disant baisse des résultats au test SAT, la hausse de l'analphabétisme, les investissements dans l'enseignement public ou le niveau des écoles privées par rapport aux écoles publiques.

HEDJERASSI Nassira, STUMPF Alexia, "Discours sur l'école en crise en France : entre médiatisation et résistance ", Revue canadienne d'éducation, 2006, vol. 29, $n^{\circ}$ 1, p. 91-108 [http://goo.gl/EY9FyC]

Cet article interroge les discours sur la crise de l'école en France à partir d'une analyse d'articles issus du quotidien français Le Monde, puis d'un examen des discours médiatisés sur l'école par des chercheurs et penseurs de l'école, et les défenseurs de l'école républicaine, et enfin la présentation critique du débat sur l'école organisé en 2003-2004. Cette recherche visait à examiner la part de construction médiatique et politique de l'expression "crise de l'école " aussi souvent convoquée que peu définie et de mesurer l'effet de contamination de cette thématique de crise de l'école dans l'opinion et les discours communs.

\section{MÉdiatisation de L’Éducation ET TRAVAIL JOURNALISTIQUE}

BARATS Christine, "Généalogie de la co-construction médiatique du "classement de Shanghai" en France. Corpus de presse et usages sociodiscursifs ", Mots. Les langages $d u$ politique, $n^{\circ}$ 102, juillet 2013, p. 67-83

L'auteure étudie, à partir d'un corpus de presse, la médiatisation du classement dit «de Shanghai » et ses usages sociodiscursifs de 2003 à 2010. La presse française a contribué à le 
co-construire comme un événement annuel et comme référence archétypale à la notion de palmarès dans le champ académique. La médiatisation du «classement de Shanghai » a ainsi participé à l'inscription de la notion de classement à l'ordre du jour du champ académique. Ce classement a fonctionné dans les discours comme un référent social avec la stabilisation de la formule classement de Shanghai. Il a porté une logique de "name and shame » en montrant du doigt les universités françaises qui apparaissaient comme ne tenant pas leur rang.

BOUCHARD Julie, «La fabrique d'un classement médiatique de l'enseignement supérieur et de la recherche: le cas du Monde de l'éducation (1976-1988) », Quaderni, 2012, $n^{\circ}$ 77, p. 25-40

Cet article traite de la production des classements médiatiques des établissements d'enseignement supérieur et de recherche, dans une perspective historique, sociologique et constructiviste. Il montre, autour de leur émergence à la fin des années 1970, et à travers le cas particulier du mensuel Le Monde de l'éducation, l'impossible neutralité de ces dispositifs fondés sur la quantification. Irréductibles à des considérations méthodologiques bien réelles, les classements médiatiques sont aussi des dispositifs politiques, journalistiques, commerciaux, organisationnels et techniques.

FORESTIER Yann, L'école, exception médiatique. La presse face aux enjeux des changements pédagogiques, thèse de doctorat en histoire contemporaine, Université de Paris IV, juin 2014, Paris IV

Cette thèse de doctorat s'interroge sur l'intensité des débats, controverses et polémiques qui, en France, abordent la question de l'école, au moyen de l'étude du discours porté par la presse écrite généraliste entre 1959 et 2008, soit au cours du demi-siècle où se rencontrent la domination de ces débats par les enjeux liés à la démocratisation de l'enseignement du second degré et l'affirmation de la presse nationale d'information générale comme instance de prescription des débats intellectuels. Basé sur l'analyse statistique d'importants échantillons sélectionnés au sein d'un corpus de 8500 articles, ce travail s'attache à relier le contenu de ces textes aux réalités dont ils prétendent rendre compte, mettant en évidence les représentations qui sont élaborées et mobilisées.

PADIOLEAU Jean-Gustave, "Systèmes d'interaction et rhétoriques journalistiques ", Sociologie du travail, 1976, $n^{\circ}$ 3, p. 256-282

Cette étude examine les journalistes spécialistes des rubriques de l'éducation nationale ; son objet premier est d'élaborer un cadre d'analyse de la production des journalistes spécialistes. La perspective d'étude choisie est celle de l'action sociale. Les représentations que les chroniqueurs universitaires se font de leurs situations et fonctions ainsi que les modalités particulières de leurs activités sont alors interprétées comme le résultat de stratégies d'action. L'auteur tente ainsi de montrer comment les « rhétoriques journalistiques » mises en œuvre par les rédacteurs dépendent des systèmes d'interaction et de pratiques sociales dont il examine les modalités d'emprise.

PONS Xavier, "Les journalistes français en éducation : une exploration typologique ", Éducation et sociétés, 2014, $n^{\circ} 33$, 1, à paraître.

Cet article étudie les fondements de l'identité professionnelle d'une catégorie d'acteurs encore très peu étudiée par la recherche: les journalistes en éducation français travaillant dans des médias nationaux. Sur la base d'une enquête qualitative par entretiens auprès de trente d'entre eux, il propose une typologie pragmatique distinguant quatre profils types de journalistes : les " experts", les «convertis », les "polyvalents» et les " outsiders». À l'opposé des discours homogénéisants sur cette catégorie d'acteurs, l'article montre ainsi que leur identité professionnelle dépend d'une série de variables que la typologie permet de condenser et qu'il convient de prendre en compte dans l'analyse des problématisations de l'action publique à l'œuvre dans les productions médiatiques. 


\section{QUelQues ÉTUdes de CAS}

RONNBERG Linda, LINDGREN Joakim, SEGERHOLM Christina, "In the Public Eye: Swedish School Inspection and Local Newspapers: Exploring the AuditMedia Relationship ", Journal of education policy, 2013, vol. 28, $n^{\circ} 2$, p. 178-197

Cet article explore la relation entre la couverture médiatique des journaux locaux suédois et les activités d'inspection de l'école. Les questions portent sur la stratégie médiatique de l'inspection, la manière dont elle est représentée, les messages envoyés, et qui prend la parole. Quatre municipalités ont été choisies pour représenter différentes contextes économiques et démographiques et avec des expériences précédentes d'inspection. Le matériau comprend des entretiens avec des membres de l'inspection et des articles de journaux. Les journaux locaux dépeignent l'inspection comme une institution légitime agissant au nom de la protection du public et, et plus encore, du consommateur éducatif. Le format utilisé par l'Inspection - un rapport succinct qui porte uniquement sur les écarts - est en lien avec le format privilégié des médias. Le renforcement de la relation étroite médias-inspection a des implications pour la gouvernance et la politique de l'éducation.

STRUYVE Charlotte, SIMONS Maarten, VERCKENS Anneleen, "Parents are not born, they are made: a critical discourse analysis of an educational magazine in Flanders (Belgium) », Journal of educational policy, 2014, vol. 29, $n^{\circ} 6$, p. $785-803$

L'article a pour étude de cas un magazine de l'éducation flamand Klasse voor Ouders (classe pour les parents). Ce magazine populaire vise à informer et communiquer avec les parents. Malgré le caractère formel et neutre revendiqué par ce magazine, les auteurs supposent qu'il joue un plus grand rôle en contribuant à la (ré)organisation du débat public. Au moyen d'une analyse critique du discours, les auteurs montrent comment les parents sont perçus comme ayant un «besoin » continu d'améliorer la qualité de leur propre pratique de parents tandis que le gouvernement se positionne comme chargé de créer un genre de "communauté d'apprentissage parental». 